\title{
Establishing a generalized polyepigenetic biomarker for tobacco smoking
}

\author{
Karen Sugden $\mathbb{B}^{1,2}$, Eilis J. Hannon $\mathbb{0}^{3}$, Louise Arseneault ${ }^{4}$, Daniel W. Belsky ${ }^{5}$, Jonathan M. Broadbent ${ }^{6}$, \\ David L. Corcoran², Robert J. Hancox', Renate M. Houts' ${ }^{7}$, Terrie E. Moffitt ${ }^{1,2,8}$, Richie Poulton ${ }^{9}$, Joseph A. Prinz ${ }^{2}$, \\ W. Murray Thomson (10), Benjamin S. Williams ${ }^{1,2}$, Chloe C. Y. Wong ${ }^{4}$, Jonathan Mill (1) ${ }^{3}$ and Avshalom Caspi ${ }^{1,2,4,8}$
}

\begin{abstract}
Large-scale epigenome-wide association meta-analyses have identified multiple 'signatures" of smoking. Drawing on these findings, we describe the construction of a polyepigenetic DNA methylation score that indexes smoking behavior and that can be utilized for multiple purposes in population health research. To validate the score, we use data from two birth cohort studies: The Dunedin Longitudinal Study, followed to age-38 years, and the Environmental Risk Study, followed to age-18 years. Longitudinal data show that changes in DNA methylation accumulate with increased exposure to tobacco smoking and attenuate with quitting. Data from twins discordant for smoking behavior show that smoking influences DNA methylation independently of genetic and environmental risk factors.

Physiological data show that changes in DNA methylation track smoking-related changes in lung function and gum health over time. Moreover, DNA methylation changes predict corresponding changes in gene expression in pathways related to inflammation, immune response, and cellular trafficking. Finally, we present prospective data about the link between adverse childhood experiences (ACEs) and epigenetic modifications; these findings document the importance of controlling for smoking-related DNA methylation changes when studying biological embedding of stress in life-course research. We introduce the polyepigenetic DNA methylation score as a tool both for discovery and theory-guided research in epigenetic epidemiology.
\end{abstract}

\section{Introduction}

Tobacco smoking is the greatest health hazard in the modern world. Smoking is associated with practically every risk factor known to impact health and with numerous clinical endpoints ${ }^{1}$. As such, uncovering methods to identify and quantify the biological mechanisms through which tobacco smoking exerts its effects is of critical public health importance.

One such mechanism is DNA methylation. Smoking is known to exact pervasive alterations on the blood epigenome $^{2-5}$. Epigenome-wide association studies (EWAS)

Correspondence: Karen Sugden (Karen.sugden@duke.edu)

'Department of Psychology and Neuroscience, Duke University, Durham, NC, USA

${ }^{2}$ Center for Genomic and Computational Biology, Duke University, Durham, NC, USA

Full list of author information is available at the end of the article. of DNA methylation have identified thousands of CpG sites differentially methylated in smokers, though the significance of these discoveries for population health science remains uncertain. If DNA methylation changes accumulate with increased exposure and attenuate with sustained abstinence, DNA methylation analysis could provide clinicians and researchers with a biomarker of smoking-associated health risk.

Some attempt has been made to capture these manifold DNA methylation differences into single, tangible biomarkers describing various aspects of smoking-related behavior and outcomes. At the simplest level, DNA methylation level at a single CpG site within $A H R R$ (described by Illumina array probe ID cg05575921) has been shown to discriminate between adult non-smokers and current-smokers ${ }^{6,7}$, and former- from neversmokers ${ }^{8}$, while other multi-probe scores have also been

\section{(c) The Author(s) 2019}

(c) (i) Open Access This article is licensed under a Creative Commons Attribution 4.0 International License, which permits use, sharing, adaptation, distribution and reproduction cc) in any medium or format, as long as you give appropriate credit to the original author(s) and the source, provide a link to the Creative Commons license, and indicate if changes were made. The images or other third party material in this article are included in the article's Creative Commons license, unless indicated otherwise in a credit line to the material. If material is not included in the article's Creative Commons license and your intended use is not permitted by statutory regulation or exceeds the permitted use, you will need to obtain permission directly from the copyright holder. To view a copy of this license, visit http://creativecommons.org/licenses/by/4.0/. 
developed to assess various phenotypes, ranging from exposure to maternal smoking ${ }^{9-11}$ to smoking-related mortality ${ }^{12}$. However, the suitability of these scores to index generalized smoking phenotypes is unknown, since these metrics are usually developed and tested in one specific population at one point in time with one specific outcome. Additionally, temporal dynamics of composite DNA methylation metrics cannot be assessed using crosssectional designs.

Longitudinal repeated-measures analysis of blood DNA methylation in smokers and non-smokers followed over time is needed to test (a) if further accumulation of exposure in smokers is reflected in accumulating changes to DNA methylation, and (b) if cessation and abstinence are associated with reversal of these changes. Finally, data are needed that observe organ system damage caused by smoking in order to test if a DNA methylation biomarker measures not just smoking behavior, but substantive health consequences of tobacco exposure.

Here, we address four major themes relevant to the relationship between tobacco smoking and DNA methylation. First, draw on published EWAS findings of smoking to construct a DNA methylation-based algorithm (Smoking methylation PolyEpigenetic Score, SmPEGS) and test its association with cross-sectional smoking phenotypes. Second, we examine the hypothesis that tobacco smoking has causal effects on DNA methylation in blood via investigation of (a) SmPEGS among twins discordant for smoking, and (b) the pattern of change over time in SmPEGS for those who either increase or cease tobacco consumption, or become nicotine dependent. In addition, we examine the relationship between SmPEGS and gene expression profiles in those who do, and do not, smoke in an attempt to uncover potential mechanistic influences of SmPEGS. Third, we analyze longitudinal repeated-measures data on lung function and periodontal disease to test if smokingassociated DNA methylation could provide a biomarker of biological damage arising from tobacco exposure. Fourth, to demonstrate a potential application of polyepigenetic scores, we conduct EWAS of adverse childhood experiences, an exposure thought to have long-term impacts on health via biological embedding. We examine the confounding effects of tobacco smoking in DNA methylation research and demonstrate the potential of utilizing the SmPEGS in lieu of observational smoking data as a covariate to reduce spurious associations in studies of the effects of early adversity on health.

We address these themes using data from two cohorts. The first is the Dunedin Longitudinal Study, a longitudinal birth cohort of 1037 individuals born in 1972-73 and followed repeatedly until the latest assessment at age38 years. Study members gave blood for DNA analysis first at age-26, and 12 years later when they were aged 38 .
The second cohort is the E-Risk longitudinal twin study, a cohort of 2232 twins (56\% MZ) born in 1994. E-Risk Study members gave blood for DNA analysis at the most recent assessment, aged 18 years.

\section{Materials and methods}

Detailed information about sampling, measurement, and statistical analysis is available in the Supplementary materials.

\section{Sample \\ Dunedin Study}

Participants were members of the Dunedin Longitudinal Study. Participants $(N=1037$; $91 \%$ of eligible births; $52 \%$ male) were all individuals born between April 1972 and March 1973 in Dunedin, New Zealand, who were eligible based on residence in the province and who participated in the first assessment at age $3^{13}$. The cohort represented the full range of socioeconomic status (SES) in the general population of New Zealand's South Island. Assessments were carried out at birth and ages 3, 5, 7, 9, $11,13,15,18,21,26,32$, and, most recently, 38 years, (95\% retention rate).

\section{E-Risk Study}

The Environmental Risk (E-Risk) Longitudinal Twin Study, tracks the development of a 1994-1995 birth cohort of 2232 British children ${ }^{14}$. Briefly, the E-Risk sample was constructed in 1999 and 2000, when 1116 families (93\% of those eligible) with same-sex 5-year-old twins participated in home-visit assessments. This sample comprised 56\% monozygotic and 44\% dizygotic twin pairs, and sex was evenly distributed within zygosity ( $49 \%$ male). The study sample represents the full distribution of socioeconomic conditions in Great Britain. Assessments were carried out at ages 5, 7, 10,12, and, most recently, 18 years ( $93 \%$ retention rate).

\section{Smoking history}

Smoking behavior in Dunedin has been assessed repeatedly between ages 15-38 years, including information about quantity smoked, cessation, and second-hand smoke exposure. In E-Risk, participants were interviewed about their smoking history at age-18 years.

\section{DNA methylation}

In Dunedin, DNA was derived from peripheral blood drawn at ages 26 and 38 years. In E-Risk, peripheral whole blood was drawn at age-18 years. In both cohorts, DNA methylation was measured using the Illumina Infinium HumanMethylation450 BeadChip ("Illumina 450K array"; Illumina, CA, USA). 


\section{Gum health}

Periodontal attachment loss was assessed at ages 26 and 38 in the Dunedin Study by calibrated dental examiners.

\section{Lung function}

Single-breath diffusing capacity for carbon monoxide (DLco/VA) was assessed at ages 26 and 38 in the Dunedin Study according to European Respiratory Society/American Thoracic Society standards using a body plethysmograph (CareFusion, Yorba Linda, CA). Measures were corrected for hemoglobin, height, smoking within an hour of assessment and $\operatorname{sex}^{15}$.

\section{Gene expression}

RNA was derived from peripheral blood drawn into PAXGene RNA tubes at age-38 in Dunedin. Expression data were generated from whole-blood RNA using the Affymetrix PrimeView Human Gene Chip (Affymetrix, CA, USA).

\section{Adverse childhood experiences (ACEs)}

The measured ACEs correspond to the ten subcategories of childhood adversity introduced by the U.S. Centers for Disease Control \& Prevention (CDC) Adverse Childhood Experiences Study; ${ }^{16}$ i.e., three types of abuse (emotional, physical, sexual), five types of household challenges (household partner violence, household substance abuse, mental illness in household, loss of a parent due to parental death, separation, or divorce, incarceration of a family member), and two types of neglect (emotional, physical).

\section{Statistical analysis}

All analyses were performed in the $\mathrm{R}$ statistical programming environment (version 3.4.2). In brief, linear regression was used to test association between dependent and independent variables, while in E-Risk, Generalized Estimating Equations (GEE) were used to account for the clustering within families.

\section{Code availability}

Code available on request from corresponding author.

\section{Results}

\section{A. Methylation polygenic scores were differentially} distributed in never-, former-, and current-smokers

We computed a smoking methylation polyepigenetic score (SmPEGS) for tobacco exposure based on the 2623 CpG probes identified by Joehanes et al. ${ }^{2,11}$ in their epigenome-wide meta-analysis of current vs. never smoking (Supplementary Table S1). We calculated SmPEGS values by multiplying the \% methylation measured at a CpG site with the effect-size estimated for that $\mathrm{CpG}$ by Joehanes et $\mathrm{al}^{2}$ and then computing the average across the set of CpGs. The resulting SmPEGSs were differentially distributed in never-, former- and currentsmokers in the Dunedin and E-Risk cohorts. In the Dunedin cohort, as compared to never-smokers $(n=405)$, former-smokers $(n=233)$ had SmPEGSs on average $0.45 \mathrm{SD}$ units higher [95\%CI 0.33-0.58] and currentsmokers $(n=165)$ had SmPEGSs on average $1.65 \mathrm{SD}$ units higher [95\%CI 1.51-1.80] $(p<0.001$ for both comparisons; Fig. 1a). In the E-Risk cohort, as compared to never-smokers $(n=1203)$, former-smokers $(n=57)$ had SmPEGSs on average $0.21 \mathrm{SD}$ units higher [95\% CI $0.02-0.40]$ and current-smokers $(n=374)$ had SmPEGSs on average 1.00 SD units higher [95\% CI 0.87-1.13] ( $p=$ 0.03 for former-smokers; $p<0.001$ for current-smokers; Fig. 1b). Supplementary Fig. S2 shows SmPEGS successfully discriminated never- from both current- and eversmokers in both Dunedin and E-Risk (AUC range from 0.77-0.93).

\section{Methylation polygenic scores tracked upwards with cumu- lative cigarette consumption}

Dunedin Study members have been interviewed repeatedly about their smoking behavior since age 15 years ${ }^{17}$. We used these prospective data to calculate the number of pack-years each Study member had smoked through the most recent interview at age-38 years. At age38 years, for Dunedin Study members who had ever smoked $(n=397)$, pack-years ranged from $0.05-38.75$ (mean $=11.71, \mathrm{SD}=8.65)$. Those who had smoked more pack-years had higher SmPEGS; each additional pack-year smoked was associated with a $0.07 \mathrm{SD}$ unit higher SmPEGS [95\% CI 0.06-0.08] $(p<0.001)$ (Fig. 1c).

Next, we turned to the 18-year-olds in the E-Risk sample. At age-18 years, for E-Risk Study members who had ever smoked $(n=431)$, pack-years ranged from 0.05-14.85 (mean=2.04 SD=1.76). Those who had smoked more pack-years had higher SmPEGS; each additional pack-year smoked was associated with a $0.1 \mathrm{SD}$ unit higher SmPEGS [95\% CI 0.05-0.15] $(p<0.001)$, similar to the 0.07 -unit increase estimated in the Dunedin data (Fig. 1d).

\section{B. Tobacco smoking has independent causal effects on DNA methylation}

Smoking and DNA methylation polygenic scores are heritable, but their association is not fully accounted for by genetic variation

Using the twin design of the E-Risk Study ${ }^{18}$, we tested genetic influences on smoking behavior. Consistent with previous research ${ }^{19}$, we find a substantial proportion of variation in pack-years smoked is under genetic influence $(\mathrm{rMZ}=0.69, \mathrm{rDZ}=0.45 ;$ additive genetic variance $=$ $48.5 \%$ [95\%CI 35.5-61.8\%]; shared environmental variance $=16.0 \% \quad[95 \% \mathrm{CI} \quad 4.1-27.3 \%] ; \quad$ non-shared 


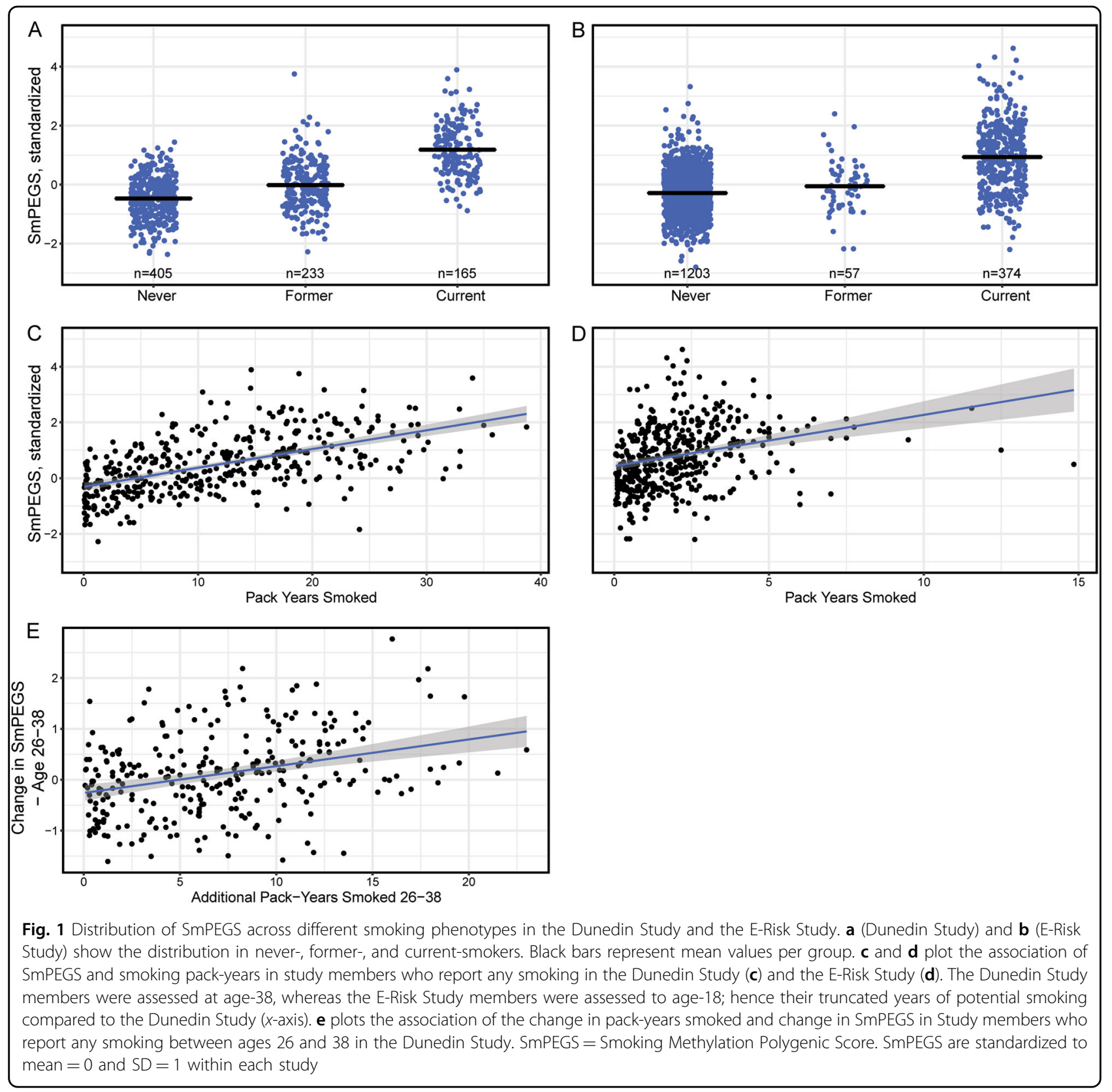

environmental variance $=35.5 \% \quad$ [95\% CI $31.8-39.7 \%$; Supplementary materials). In parallel, we found that a substantial proportion of variation in SmPEGS is under genetic influence $(\mathrm{rMZ}=0.87, \mathrm{rDZ}=0.52 ;$ additive genetic variance $=70.9 \%$ [95\% CI 60.4-82.6\%]; shared environmental variance $=16.0 \% \quad[95 \%$ CI $4.3-26.5 \%]$; non-shared environmental variance $=13.1 \% \quad[95 \%$ CI 11.7-14.6\%]).

To test if genetic and shared environmental factors might confound associations between tobacco exposure and SmPEGS values, we studied differences between twins.
Specifically, we parsed the effect of pack-years smoked on SmPEGS into between-twin and within-twin-pair effects (Supplementary materials). Within-twin-pair differences in pack-years among both DZ and MZ twins were significantly associated with differences in SmPEGS, such that the co-twin who smoked more had higher SmPEGS $(b=0.18$, [95\% CI 0.10-0.25] $p<0.001)$. We found a similar pattern when the analysis was repeated using only MZ twins $(b=0.09$, [95\% CI 0.02-0.16] $p=0.01)$, indicating the association could not be fully explained by shared family-wide environmental or genetic factors. 
Study members who smoked additional pack-years between ages 26 and 38 experienced increase in their methylation polygenic scores

Dunedin Study members provided DNA for analysis of methylation at two time points, ages 26 and 38 . To isolate the effect of accumulating tobacco exposure on changes in the methylome, we utilized the repeated-measures of tobacco exposure and SmPEGS in the Dunedin Study. For Study members who accumulated pack-years between ages 26 and $38(n=280)$, we regressed change in SmPEGS on the number of additional pack-years smoked. Compared to their age- 26 baseline, each additional pack-year smoked between ages 26 and 38 years was associated with a 0.05 SD unit increase in SmPEGS [95\% CI 0.03-0.07] (Fig. 1e). Accumulating tobacco exposure is reflected in corresponding SmPEGS change.

Dunedin Study members who quit smoking between methylation measurements at ages 26 and 38 experienced a relative decline in their methylation polygenic scores

Figure 2 describes SmPEGS changes over time in four groups; individuals who have never smoked, those who quit smoking by age-26, those who quit between the two assessments at ages 26 and 38, and those who were still smoking at age-38. For individuals who had never smoked or quit smoking by age-26, SmPEGS stayed relatively static between ages 26 and 38 (mean SD unit change $=$ -0.04 [95\% CI $-0.1-0.01]$ for never-smokers, and -0.04 [95\% CI $-0.19-0.11]$ for those who quit by age-26). Those individuals still smoking at age-38 experienced an increase in their SmPEGS compared to age-26 (mean SD unit change $=0.49[95 \%$ CI $0.36-0.62]$ ). However, those who ceased smoking between ages 26 and 38 experienced a relative decline in their SmPEGS over the same period (mean SD unit change $=-0.25[95 \% \mathrm{CI}-0.36$ to -0.14$]$ ); compared to the never-smokers, SmPEGS declined 0.21 SD units [95\% CI 0.09-0.32]) over this period (Fig. 2). Ceasing tobacco use is reflected in corresponding SmPEGS recovery.

Dunedin Study members who are nicotine dependent have higher methylation polygenic scores than non-dependent smokers, and these scores are higher even after taking into account density of tobacco consumption

In addition to the analysis of changes in SmPEGS related to changes in smoking behavior over time, we conducted analysis of changes in SmPEGS related to changes in nicotine dependence using the Fagerstrom Test of Nicotine Dependence (FTND) at ages 26 and $38^{17,20}$. This instrument is designed to test dependence on nicotine by assessing, for example, criteria related to frequent urges, valuing smoking most after abstinence, and smoking density. Within those reporting current smoking at age-26, nicotine-dependent Study members had SmPEGS 0.43 SD units [95\% CI 0.20-0.65] higher than those not meeting criteria for nicotine dependence. Similarly, at age-38, nicotine-dependent Study Members had SmPEGS 0.61 SD units [95\% CI 0.34-0.88] higher than non-dependent Study Members. For individuals who were never dependent, SmPEGS stayed relatively stable between ages 26 and 38 (mean SD unit change $=-0.04$ [95\% CI -0.12-0.05]). Those individuals who were dependent only at age- 26 experienced a slight decline in their SmPEGS between ages 26 and 38 (mean SD unit change $=-0.12[95 \% \mathrm{CI}-0.36-0.11]$ ). Those individuals who were dependent at either age-38 only, or at both ages, experienced relative increases in their SmPEGS between ages 26 and 38 (mean SD unit change $=0.58$ [95\% CI $0.25-0.91$ ] for age-38 only dependent individuals, and 0.60 [95\% CI 0.33-0.87] for those who are dependent at

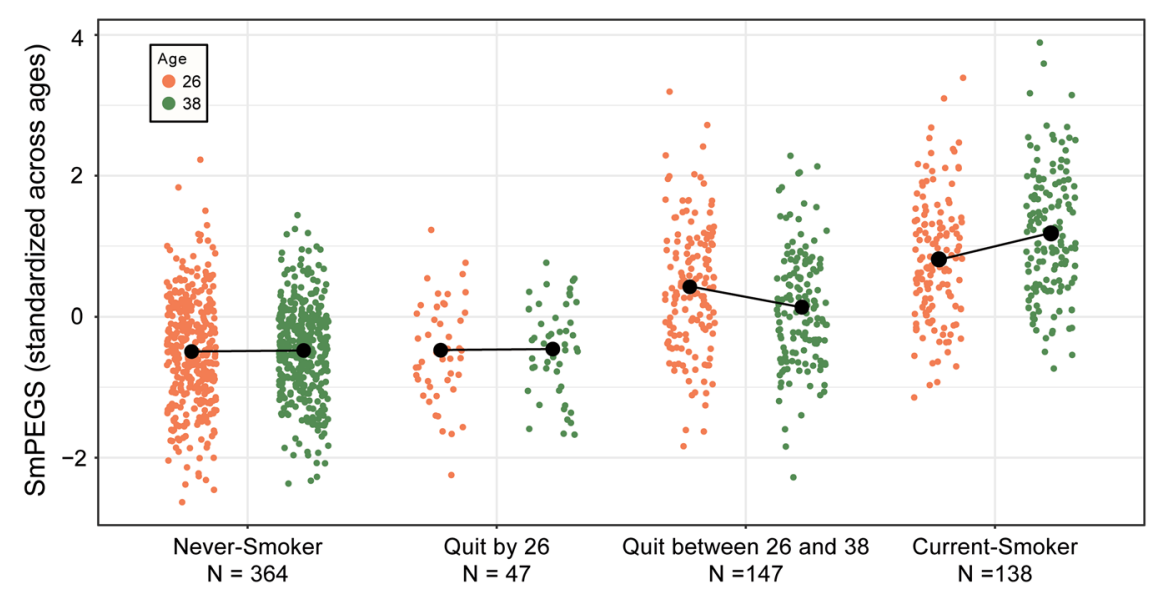

Fig. 2 Distribution of SmPEGS in the Dunedin Study at two time points across 12 years as a function of smoking history at age-38. The figure shows Smoking methylation Polygenic Score (SmPEGS) were reduced for those who ceased to smoke between the two DNA methylation assessments at ages 26 and 38. Black points represent means within each group connected by lines across the two assessment phases 
both ages). Compared to smokers who do not meet diagnostic criteria at either age, SmPEGS increased 0.62 SD units [95\% CI 0.30-0.93] and 0.63 SD units [95\% CI 0.40-0.87] in these two groups, respectively (Supplementary Fig. S3). Because diagnosis of nicotine dependence can rely heavily on density of smoking (dependent smokers have on average 8.73 [95\% CI 6.96-10.50] more pack-years by age-38 than non-dependent smokers), and because we have demonstrated SmPEGS increase with cumulative tobacco consumption, we further tested whether these associations with FTND were accounted for by accelerated accumulation of tobacco between the two ages. Compared to smokers who do not meet diagnostic criteria at either age, SmPEGS increased 0.37 SD units [95\% CI $0.02-0.73]$ and $0.35 \mathrm{SD}$ units [95\% CI 0.05-0.66] for individuals who were dependent at either age-38 only, or at both ages, respectively, when change in number of pack-years between age- 26 and 38 was included as a covariate in the analysis. Diagnosis of nicotine dependence predicts inter-age increases in SmPEGS in these two groups even after controlling for accumulation in pack-years between 26 and 38, suggesting the SmPEGS indexes physical aspects of smoking behavior above and beyond volume of cigarette consumption.

DNA methylation differences identified in epigenome-wide association studies of smoking relate to gene expression differences in both smokers and non-smokers

DNA methylation has been shown to play a role in mediating gene expression, both directly and indirectly $^{21-24}$. We tested whether the SmPGS, a composite measure of 2623 smoking-related DNA methylation sites, predicts differences in global gene expression by conducting transcriptome-wide analysis of the SmPGS in Dunedin study participants at age-38. This analysis identified 143 differentially expressed probesets within 98 genes (Supplementary Table S2.). Pathway analysis revealed that networks related to cellular movement, immune cell trafficking, hematological system development and function, cell-mediated immune response, and inflammatory response were the most significantly enriched for these genes (Supplementary Table S3.).

We then asked whether individual DNA methylation probes were responsible for these associations by deconstructing the SmPGS. We conducted integrative genomic analysis by testing correlations between methylation levels at the 2623 DNA methylation probes included in the SmPGS and expression levels of the 143 gene expression probesets. To investigate any possible underlying biological relationship between these correlated methylation probes and expression probesets, we conducted this analysis separately in two groups of individuals: those who currently smoke and those who have never smoked. This rationale deems that if a relationship between DNA methylation and gene expression is not dependent solely on shared influence of smoking, then it should be observable both in individuals who have never smoked as well as in those who have. If the relationship is driven by smoking, then it would be observable in just one of these groups. Of the 375,089 correlations tested, 108 were significant after Bonferroni correction $\left(p<1.33 \times 10^{-7}\right)$ in both non-smokers and smokers, representing 47 unique DNA methylation probes and 22 unique gene expression probesets in 14 genes (Supplementary Table S4.).

Most of the correlations between DNA methylation probes and expression probesets identified above describe trans relationships; only one of the 47 DNA methylation probes was cis (defined as $250 \mathrm{~Kb}$ up- or down-stream of the start of a gene) to a correlated probeset, cg26724967 located 124 bp upstream of the start of IL32. This DNA methylation probe is negatively correlated with four probesets indexing IL32 gene expression (Fig. 3). IL32 is an inflammatory cytokine that has been previously associated with COPD and smoking-related damage to the lungs ${ }^{25,26}$. Cis relationships between DNA methylation and IL32 expression have been previously observed in smokers $^{2}$, but it is not clear that the relationship is independent of smoking; here we document cis relationships in non-smokers, which suggests the potential for a causal biological effect of proximal DNA methylation on IL32 expression independent of smoking. IL32 is an excellent candidate for understanding the biological impact of DNA methylation on disease risk.

As a sensitivity analysis, we entertained the possibility that methylation-expression correlations among nonsmokers arise because of passive exposure to secondhand smoking that exerts similar effects to the direct consumption of tobacco products. We repeated the correlation analyses in the never-smoker group while controlling for self-reports of exposure to second-hand smoke (for example, in the home or workplace). The magnitude and direction of correlations between DNA methylation probes and gene expression probesets were comparable to the non-adjusted correlation statistics (spearman's $r h o=0.99, p<0.001$, Supplementary Table S4), suggesting the observed associations reflect biological relationships not driven by underlying passive tobacco consumption.

\section{C: Methylation polygenic score changes between 26 and 38 years track smoking-associated damage to lungs and gums}

Our longitudinal analysis in the Dunedin cohort supports the hypothesis that SmPEGS tracks the accumulation of tobacco exposure and SmPEGS becomes attenuated over time among those who quit smoking. An important question, therefore, is whether SmPEGS 


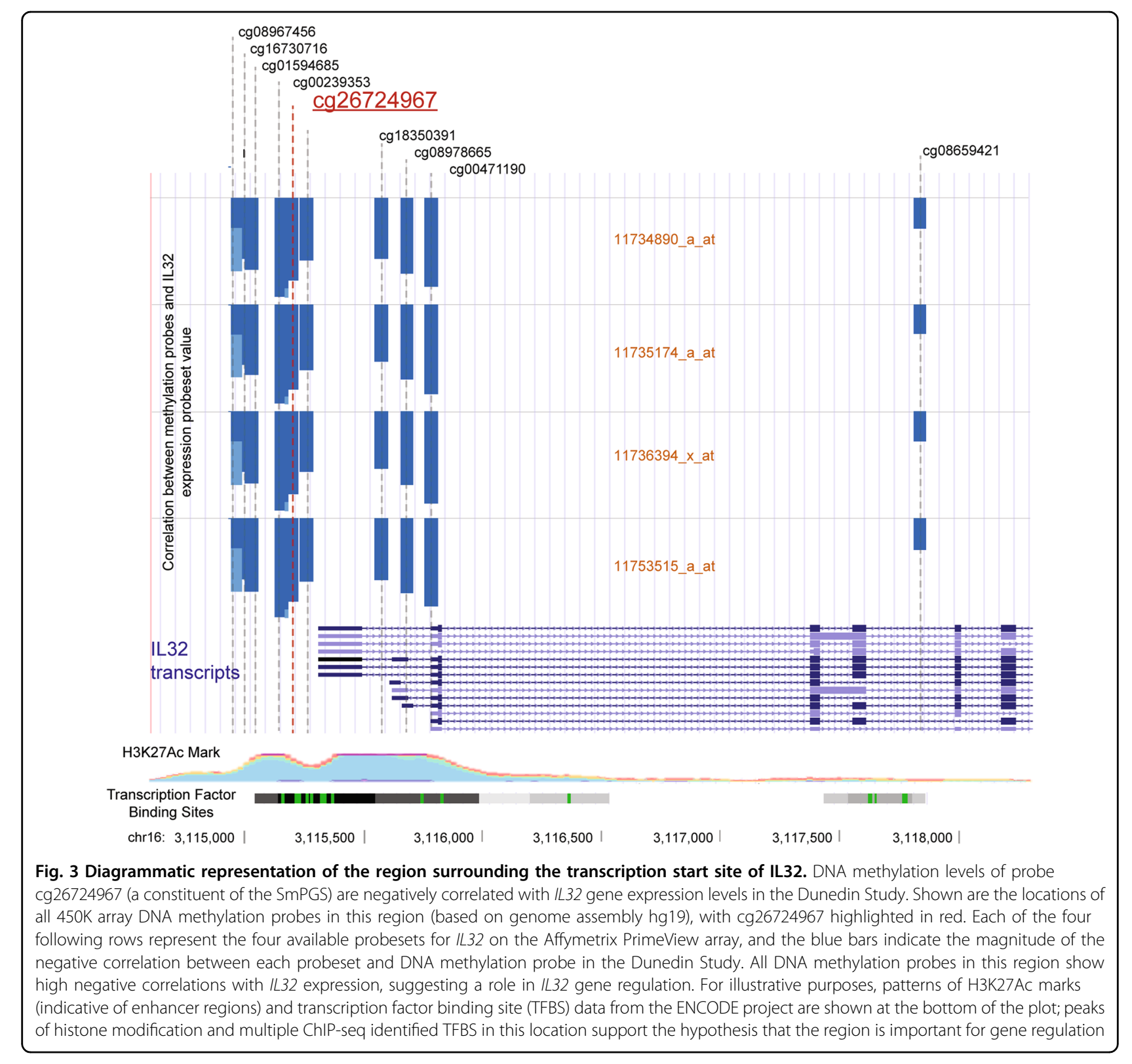

reflects processes of smoking-associated physiological damage and recovery.

We considered damage to two systems proximate to tobacco exposure: the lungs and the gums. Our analysis of lung and gum damage used longitudinal data collected at the same time as DNA methylation. Lung function was measured at age- 26 and 38 years using the carbon monoxide transfer coefficient DLco/VA (in $\mathrm{mL} / \mathrm{min} / \mathrm{mmHg} / \mathrm{L}$ ), an index of the efficiency of alveolar transfer of carbon monoxide; lower values index worse lung function. Gum health was measured at ages 26 and 38 years as periodontal attachment loss in $\mathrm{mm}$; higher values index worse gum health.

\section{Lung function}

Study members who smoked at ages 26 and 38 years suffered damage to their lungs. Current-smokers at age26 had DLco/VA measures 0.28 units lower than neversmokers, and at age-38 current-smokers had measures 0.83 units lower than never-smokers. Longitudinally, we find that within-individual changes in pack-years accumulation tracked with within-individual changes in lung function. Among those who had ever smoked, each additional pack-year smoked was associated with a 0.03SD unit decline in DLco/VA [95\%CI 0.01-0.05] (Fig. 4a). In parallel, we tested whether study members' SmPEGS was associated with lung damage. Each additional 

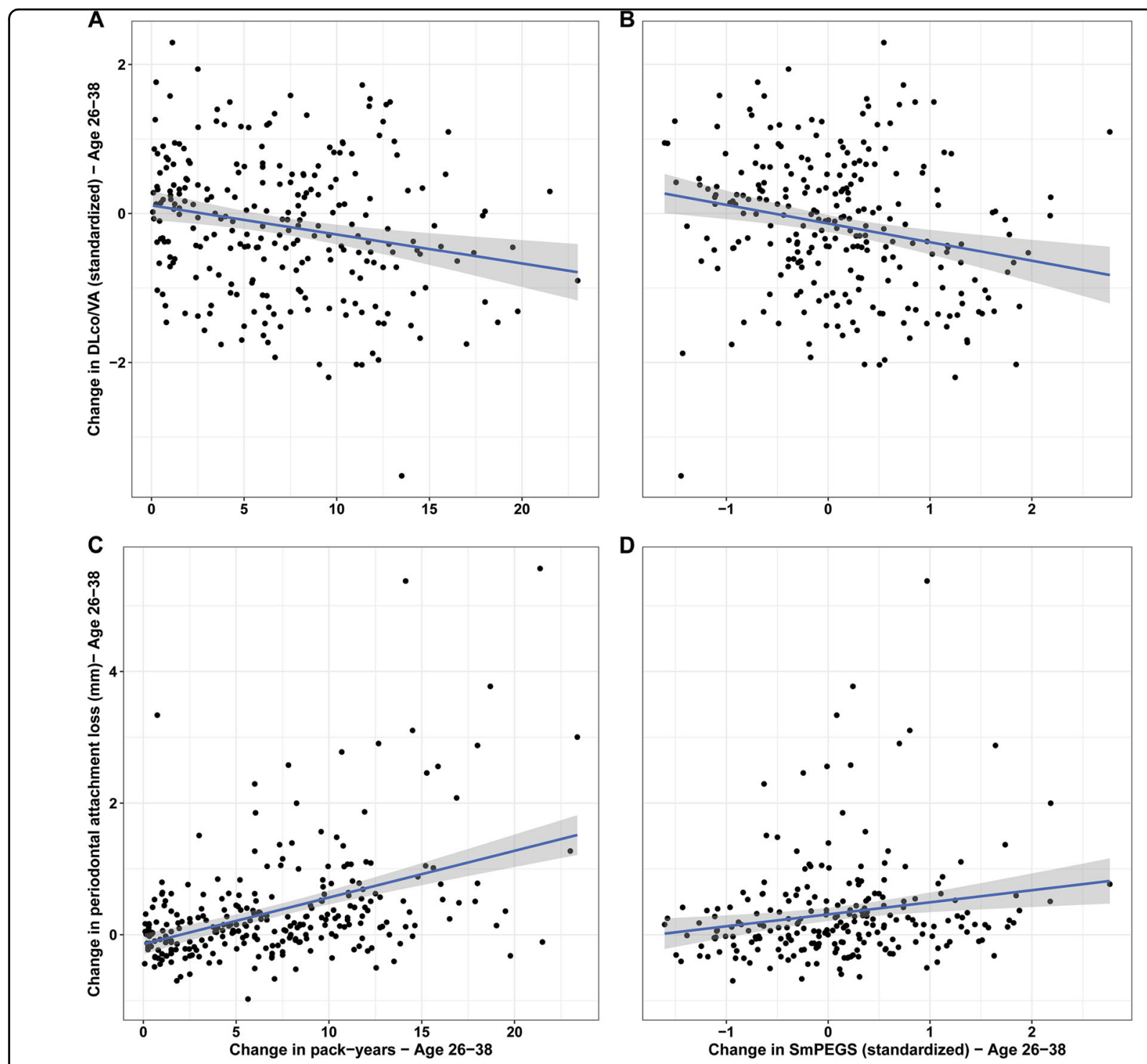

Fig. 4 Association between change in pack-years smoked or SmPEGS and change in smoking-associated damage to lungs and gums between ages 26 and 38 in the Dunedin Study. (a) and (c) show change in pack-years versus change in lung function and peridontal attachement loss, repectively. (b) and (d) show change in SmPEGS and change in lung function and periodontal attachment loss, respectively. The analysis is restricted to study members who smoked between ages 26 and 38 years

SmPEGS SD unit increase was associated with a 0.23-SD unit decline in DLco/VA [95\% CI 0.10-0.36] (Fig. 4b).

\section{Gum health}

Study members who smoked at ages 26 and 38 years suffered damage to their gums. Current-smokers at age26 had $0.13 \mathrm{~mm}$ more periodontal attachment loss than never-smokers, and at age-38 current-smokers had $0.77 \mathrm{~mm}$ more periodontal attachment loss than neversmokers. Longitudinally, we find that within-individual changes in pack-years accumulation tracked with withinindividual changes in periodontal attachment loss. Among those who had ever smoked, each additional pack-year smoked was associated with $0.07 \mathrm{~mm}$ of additional periodontal attachment loss [95\% CI 0.05-0.08] (Fig. 4c). In parallel, we tested whether study members' SmPEGS was associated with gum damage. Each additional SmPEGS SD unit increase was associated with 0.17 millimeters of additional periodontal attachment loss [95\% CI 0.06-0.28] (Fig. 4d).

These findings suggest that not only does the SmPEGS track smoking, but also smoking-related damage to biological systems. This damage is detectable external to the tissue of clinical interest, namely the lungs and gums. The 
SmPEGS, measurable in a blood sample, may serve as a suitable proxy when invasive or complicated testing are not feasible.

\section{D: Cigarette smoking confounds associations between psychosocial risk factors and DNA methylation}

Adverse experiences in childhood are associated with increased morbidity and early mortality ${ }^{16}$. Epigenetic modifications are thought to play a role in this apparent "biological embedding" of risk ${ }^{27-29}$. However, adverse childhood experiences (ACEs) are also associated with smoking behavior ${ }^{30}$, making it difficult to tease apart epigenetic associations with early stress from the effects of smoking on the epigenome ${ }^{31}$.

We conducted EWAS of reports of ACEs in the Dunedin and E-Risk studies. In both cohorts, we measured ACEs as the sum of exposure to ten types of childhood experiences. ACEs predicted differential DNA methylation at ten probes in the Dunedin Study and seven in the E-Risk Study. However, each one of these probes was associated with smoking in our Dunedin and E-Risk studies, and in the study by Joehanes et al. $^{2}$. After statistical control for number of pack-years smoked, no probes remained statistically significantly associated with ACEs in the Dunedin Study and only one probe (cg25949550) remained significant in the E-Risk Study (Supplementary Table S5).

We further tested if including the SmPEGS as a covariate would yield a similar pattern of attenuation to that observed when including smoking history as a covariate. We found that comparable statistical control for smoking could be achieved using the SmPEGS (Fig. 5). Statistical control for smoking history is vital when conducting epigenetic analyses of ACEs, and if a study lacked observed smoking history data, the SmPEGS could serve as a suitable proxy control.

\section{Discussion}

We demonstrated that DNA methylation patterns in whole blood are associated with tobacco smoking, and that composite scores comprising specific DNA methylation sites (SmPEGS) can be utilized to index smoking behavior. SmPEGS are higher in smokers than nonsmokers, are observable in both middle-aged individuals (Dunedin Study members) and young adults (E-Risk Study members), increase with additional tobacco consumption, and subside upon smoking cessation. In parallel with smoking, changes in SmPEGS over time index changes in lung function and gum health. Lastly, tobacco smoking confounds DNA methylation studies of psychosocial risk, and this can be accounted for by introducing either observed smoking history or SmPEGS as a statistical control.
There are a number of implications of SmPEGS. First, analysis of twins in the E-Risk Study shows that, while there is substantial familiality to SmPEGS, the association with smoking cannot be fully accounted for by either genetics or shared environmental effects. This is in line with several smaller twin studies that have focused on DNA methylation at individual probe sites ${ }^{32,33}$, and other research that demonstrates that while methylation Quantitative Trait Loci (mQTLs) for smoking-related probes exist, a substantial amount are independent of genetic correlates ${ }^{34}$. These observations combined suggest that smoking might elicit effects on DNA methylation independent of background risk associated with an individual's genetic or environmental propensity for smoking.

Second, changes in SmPEGS parallel smoking behavior changes; Dunedin Study members who continued to smoke between age-26 and age-38 saw a corresponding increase in their SmPEGS between assessments, while those who ceased smoking over the same period saw a relative decline in their SmPEGS. Dynamic responses of DNA methylation levels to changes in smoking behavior are of particular significance to public health; if DNA methylation changes represent clinically relevant indicators of damage arising from tobacco use, then their reversal after ceasing tobacco use suggests the potential for biological recovery. Previous studies have demonstrated that CpG site-specific DNA methylation has the potential for long-term recovery via time-since-quit models of analysis ${ }^{2,5}$, although the results are not consistent $^{4,8,35,36}$. Here, using within-person changes in smoking behavior mapped to within-person changes in SmPEGs, we show that there is potential for recovery at the aggregate level.

Third, a number of DNA methylation sites that comprise SmPEGS predict corresponding changes in gene expression. These differentially expressed genes are found in pathways relating to inflammation, immune response, and cellular trafficking, indicating smoking-induced DNA methylation differences might exert an effect via inflammatory pathways. Moreover, a proportion of these correlations are observed in those who do not smoke, suggesting these DNA methylation-gene expression relationships are not artefacts of smoking behavior. That these DNA methylation sites were initially identified through differential methylation patterns in response to smoking gives rise to possible routes by which smoking exerts biological damage at the molecular level. Of particular interest is the DNA methylation probe cg26724967, negatively correlated in cis to IL32 expression. IL32 is a relatively recently discovered cytokine, and previous research has shown that IL 32 mRNA and protein levels in serum and lung tissue are higher in smokers with 

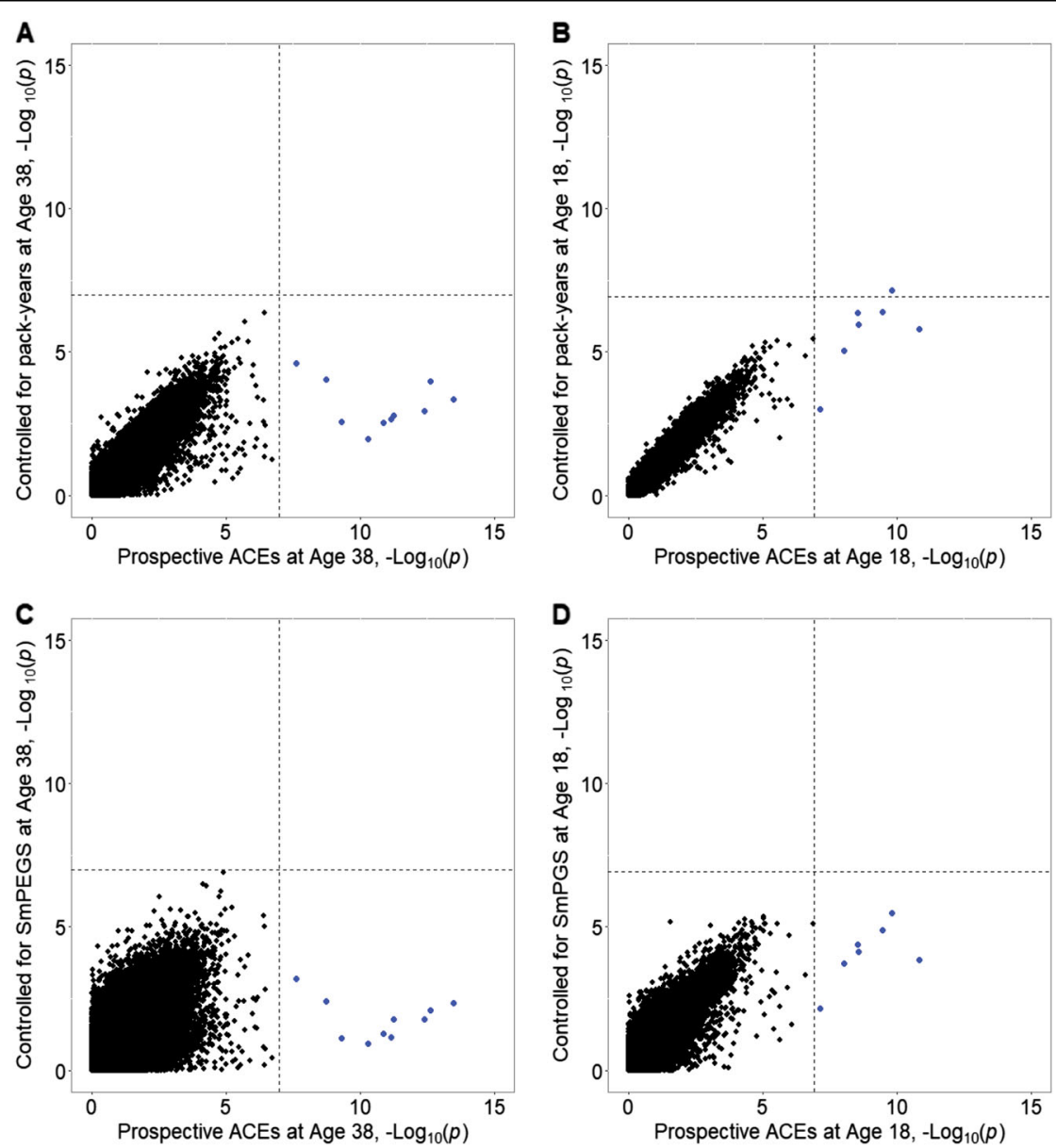

Fig. 5 Cigarette smoking confounds associations between psychosocial risk factors and DNA methylation. $\mathbf{a}, \mathbf{b}$ show the scatterplot of the $-\log _{10}(p)$ values from an epigenome-wide association studies (EWAS) of adverse childhood experiences (ACEs; $x$-axis) plotted against -Log ${ }_{10}(p)$ from an EWAS of ACEs controlling for pack-years smoked at age-38 in the Dunedin Study (a) and age-18 in the E-Risk Study (b). c, d show the same scatterplot but substituting SmPEGS at age-38 in Dunedin (c) and age-18 in E-Risk (d) for pack-years smoked. Dashed lines represent the genomewide significant cutoff levels. Blue points represent probes significant in the EWAS of ACEs. Had EWAS hits for ACEs remained significant after controlling for smoking $(\mathbf{a}, \mathbf{b})$ or for Smoking methylation Polygenic Score (SmPEGS) (c, d), the blue points would appear in the upper right-hand quadrant. In both cohorts, controlling for smoking or using the SmPEGS attenuates the association between ACEs and DNA methylation to nonsignificance; hence the blue points appear in the lower right-hand quadrant

chronic pulmonary obstructive disease (COPD) than nonsmoking COPD patients, non-COPD smokers, and healthy controls ${ }^{25,26}$. IL32 is an excellent candidate biomarker for smoking-induced damage to health, and warrants further investigation.

Fourth, changes in SmPEGS index changes in lung function and gum health over time; these changes are comparable to those predicted by changes in amount of cigarettes smoked over the same period. This suggests SmPEGS can be utilized to index damage to distal biological systems (in this case, the lungs and gums) without necessitating collection of said tissue. Evidence exists that some of these smoking-sensitive DNA methylation sites can also be identified in lung tissue ${ }^{37,38}$, and further research on the link between DNA methylation in blood and damage to distal biological systems could uncover novel biomarkers of health ${ }^{39}$.

The final implication concerns future study design ${ }^{40,41}$. Epigenome-wide association studies strive to remove effects of confounding covariates ${ }^{42}$ when the confounding factor is robustly associated with (a) the exposure/phenotype under test, and with (b) differential DNA methylation. These two points are relevant in studies of psychosocial stress, which are vigorously interrogating how "stress gets under the skin". A difficulty is that individuals who have higher levels of stress smoke more ${ }^{30}$, and smoking itself elicits differential effects on the epigenome. Here, we show that in the case of adverse childhood experiences (ACEs), smoking confounds tests of the association with DNA methylation. When testing 
associations between DNA methylation and a phenotype known to be also associated with smoking (e.g., schizophrenia, alcoholism, hypertension ${ }^{43,44}$ ), statistical control for smoking behavior should be introduced. Where a subject's smoking history is undetermined or potentially unreliable ${ }^{7}$, SmPEGS can be utilized successfully in its place. Some researchers have started to use polyepigenetic scores to index smoking, but they are developing these scores in idiosyncratic and sample specific ways. This is likely to impede the systematic accumulation of knowledge. Thus, here we have developed a standard methodology for creating polyepigenetic scores. Importantly, this framework of score construction can be extended to any measure associated with both an exposure and DNA methylation so as to generate proxy controls for confounders where necessary.

There are some caveats. First, we took a conservative approach to constructing SmPEGS by utilizing only the 2623 probes that reached genome-wide significance $(p<$ $1 \times 10-07)$ in Joehanes et al. $^{2}$. While this approach of restricting candidate markers to those with association $p$ value less than a defined threshold is common in the polygenic scoring community ${ }^{45,46}$, it is possible we omitted some biologically relevant variation in smokingrelated DNA methylation not captured by this list of probes. However, as shown in Supplementary Materials (Supplementary Table S6), we also constructed a score utilizing all 18,760 probes that reached False Discovery Rate (FDR)-level significance in Joehanes et al. $^{2}$. This score predicted smoking no better than the SmPEGS. In contrast to the polyepigenetic approach, a primary focus of previous biomarker development has been the single CpG probe cg05575921, located within the gene AHRR. Indeed, consistent with prior reports, methylation beta levels of this one probe do an excellent job of discriminating never-, former-, and current-smokers and is associated with cumulative smoking exposure in our data $^{6,7,47}$ (Supplementary Table S6). Reported associations between cg05575921 and smoking are not always consistent; for example, evidence of rebound to non-smoking levels after quitting is conflicting ${ }^{35,36,48}$, and evidence of association with dependence-associated behavior is lacking $^{6}$. To this end, it is possible that a polyepigenetic approach to assessing smoking dynamics could better capture divergent underlying biological correlates of smoking, such as nicotine craving, that a single DNA methylation signal does not. Second, we constructed SmPEGS in blood DNA; we are unable to verify whether SmPEGS are generalizable across different tissue types. It is likely that the tissue specificity of DNA methylation levels varies across the range of probes used to construct the SmPEGS ${ }^{49}$. We encourage the validation of crosstissue applicability when substrates other than blood are used. That said, given that most population-based assessments of DNA methylation are made using DNA from blood, the algorithm for construction of SmPEGS described herein has broad-scale utility for the majority of researchers. Third, we borrowed the idea of a polyepigenetic score from the methods employed by the GWAS community, and we assume that the effects are additive. As in this cognate area ${ }^{46}$, this assumption will require validation and interrogation.

In conclusion, we describe the construction of a DNA methylation composite score that indexes smoking behavior, predicts damage to biological systems known to be affected by smoking, is correlated with changes in gene expression, and can be utilized as a proxy smoking-history measure in situations where observational smoking data are unavailable.

\section{Acknowledgements \\ The Dunedin Longitudinal Study is funded by the New Zealand Health Research Council, the New Zealand Ministry of Business, Innovation, and Employment, the National Institute on Aging (AG032282), and the Medical Research Council (MR/P005918/1). The E-Risk Study is funded by the Medical Research Council (G1002190) and the National Institute of Child Health and Human Development (HD077482). Additional support was provided by a Distinguished Investigator Award from the American Asthma Foundation to Dr. Mill, and by the Jacobs Foundation and the Avielle Foundation. Dr. Arseneault is the Mental Health Leadership Fellow for the U.K. Economic and Social Research Council. Dr. Belsky is a Jacobs Foundation Fellow. This work used a high-performance computing facility partially supported by grant 2016- IDG-1013 ("HARDAC + : Reproducible HPC for Next-generation Genomics") from the North Carolina Biotechnology Center. Illumina DNA methylation data are accessible from the Gene Expression Omnibus (accession code: \\ GSE105018). We thank the Dunedin and E-Risk Study members, research staff, and Dunedin Study founder Phil Silva.}

\section{Author details}

${ }^{1}$ Department of Psychology and Neuroscience, Duke University, Durham, NC, USA. ${ }^{2}$ Center for Genomic and Computational Biology, Duke University, Durham, NC, USA. ${ }^{3}$ University of Exeter Medical School, University of Exeter, Exeter, UK. ${ }^{4}$ Social, Genetic, and Developmental Psychiatry Research Centre, Institute of Psychiatry, Psychology, and Neuroscience, King's College London, London, UK. ${ }^{5}$ Department of Epidemiology \& Butler Aging Center, Columbia University Mailman School of Public Health, New York, NY, USA. 'Department of Oral Sciences, University of Otago, Dunedin, New Zealand. ${ }^{7}$ Department of Preventive and Social Medicine, Dunedin School of Medicine, University of Otago, Dunedin, New Zealand. ${ }^{8}$ Department of Psychiatry and Behavioral Sciences, Duke University School of Medicine, Durham, NC, USA. ${ }^{9}$ Dunedin Multidisciplinary Health and Development Research Unit, University of Otago, Dunedin, New Zealand

Conflict of interest

The authors declare that they have no conflict of interest.

\section{Publisher's note}

Springer Nature remains neutral with regard to jurisdictional claims in published maps and institutional affiliations.

Supplementary Information accompanies this paper at (https://doi.org/ 10.1038/s41398-019-0430-9).

Received: 15 November 2018 Revised: 17 January 2019 Accepted: 24 January 2019

Published online: 15 February 2019 


\section{References}

1. U.S. Department of Health and Human Services, Centers for Disease Control and Prevention, National Center for Chronic Disease Prevention and Health Promotion, Office on Smoking and Health. The Health Consequences of Smoking-50 Years of Progress: A Report of the Surgeon General (U.S. Department of Health and Human Services, Atlanta, GA, 2014).

2. Joehanes, R. et al. Epigenetic signatures of cigarette smoking. Circ. Cardiovasc Genet. 9, 436-447 (2016).

3. Gao, X., Jia, M., Zhang, Y., Breitling, L. P. \& Brenner, H. DNA methylation changes of whole blood cells in response to active smoking exposure in adults: a systematic review of DNA methylation studies. Clin. Epigenetics. 7, 113 (2015).

4. Tsaprouni, L. G. et al. Cigarette smoking reduces DNA methylation levels at multiple genomic loci but the effect is partially reversible upon cessation. Epigenetics 9, 1382-1396 (2014).

5. Zeilinger, S. et al. Tobacco smoking leads to extensive genome-wide changes in DNA methylation. PLOS ONE 8, e63812 (2013).

6. Philibert, R. et al. Dose response and prediction characteristics of a methylation sensitive digital PCR assay for cigarette consumption in adults. Front. Genet. 9, 137 (2018).

7. Andersen, A. M., Philibert, R. A., Gibbons, F. X., Simons, R. L. \& Long, J. Accuracy and utility of an epigenetic biomarker for smoking in populations with varying rates of false self-report. Am. J. Med Genet. B Neuropsychiatr. Genet. 174, 641-650 (2017)

8. Shenker, N. S. et al. DNA methylation as a long-term biomarker of exposure to tobacco smoke. Epidemiology 24, 712-716 (2013).

9. Ladd-Acosta, C. et al. Presence of an epigenetic signature of prenatal cigarette smoke exposure in childhood. Environ. Res. 144(Pt A), 139-148 (2016).

10. Reese, S. E. et al. DNA methylation score as a biomarker in newborns for sustained maternal smoking during pregnancy. Environ. Health Perspect. 125 760-766 (2017).

11. Richmond, R. C., Suderman, M., Langdon, R., Relton, C. L. \& Davey Smith, G. DNA methylation as a marker for prenatal smoke exposure in adults. Int J. Epidemiol. 47, 1120-1130 (2018).

12. Zhang, Y. et al. Smoking-associated DNA methylation biomarkers and their predictive value for all-cause and cardiovascular mortality. Environ. Health Perspect. 124, 67-74 (2016)

13. Poulton, R., Moffitt, T. E. \& Silva, P. A. The Dunedin Multidisciplinary Health and Development Study: overview of the first 40 years, with an eye to the future. Soc. Psychiatry Psychiatr. Epidemiol. 50, 679-693 (2015).

14. Moffitt, T. E., Team ERS. Teen-aged mothers in contemporary Britain. J. Child Psychol. Psychiatry 43, 727-742 (2002)

15. Graham, B. L. et al. 2017 ERS/ATS standards for single-breath carbon monoxide uptake in the lung. Eur. Respir. J. 49, 1600016 (2017). https://doi.org/10.1183/ 13993003.00016-2016.

16. Felitti, V. J. et al. Relationship of childhood abuse and household dysfunction to many of the leading causes of death in adults. The Adverse Childhood Experiences (ACE) Study. Am. J. Prev. Med. 14, 245-258 (1998).

17. Belsky, D. W. et al. Polygenic risk and the developmental progression to heavy, persistent smoking and nicotine dependence: evidence from a 4-decade longitudinal study. JAMA Psychiatry 70, 534-542 (2013).

18. Hannon, E. K. O. et al. Characterizing genetic and environmental influences on variable DNA methylation using monozygotic and dizygotic twins. PLoS Genet. 14, e1007544 (2018).

19. Polderman, T. J. et al. Meta-analysis of the heritability of human traits based on fifty years of twin studies. Nat. Genet. 47, 702-709 (2015).

20. Fagerstrom, K. O. Measuring degree of physical dependence to tobacco smoking with reference to individualization of treatment. Addict. Behav. 3, 235-241 (1978).

21. Boyes, J. \& Bird, A. DNA methylation inhibits transcription indirectly via a methyl-CpG binding protein. Cell 64, 1123-1134 (1991).

22. Jaenisch, R. \& Bird, A. Epigenetic regulation of gene expression: how the genome integrates intrinsic and environmental signals. Nat. Genet. 33(Suppl), 245-254 (2003)

23. Wan, J. et al. Characterization of tissue-specific differential DNA methylation suggests distinct modes of positive and negative gene expression regulation. BMC Genom. 16, 49 (2015).
24. Anastasiadi, D., Esteve-Codina, A. \& Piferrer, F. Consistent inverse correlation between DNA methylation of the first intron and gene expression across tissues and species. Epigenetics Chromatin. 11, 37 (2018).

25. Gasiuniene, E., Lavinskiene, S., Sakalauskas, R. \& Sitkauskiene, B. Levels of IL-32 in serum, induced sputum supernatant, and bronchial lavage fluid of patients with chronic obstructive pulmonary disease. COPD 13, 569-575 (2016).

26. Calabrese, F. et al. IL-32, a novel proinflammatory cytokine in chronic obstructive pulmonary disease. Am. J. Respir. Crit. Care Med. 178, 894-901 (2008).

27. Suderman, M. et al. Childhood abuse is associated with methylation of multiple loci in adult DNA. BMC Med Genom. 7, 13 (2014).

28. Vaiserman, A. M. Epigenetic programming by early-life stress: Evidence from human populations. Dev. Dyn. 244, 254-265 (2015).

29. Klengel, T. \& Binder, E. B. Epigenetics of stress-related psychiatric disorders and gene $x$ environment interactions. Neuron 86, 1343-1357 (2015).

30. Anda, R. F. et al. Adverse childhood experiences and smoking during adolescence and adulthood. JAMA 282, 1652-1658 (1999).

31. Marzi, S. J. et al. Analysis of DNA methylation in young people: Limited evidence for an association between victimization stress and epigenetic variation in blood. Am. J. Psychiatry 175, 517-529 (2018).

32. Allione, A. et al. Novel epigenetic changes unveiled by monozygotic twins discordant for smoking habits. PLOS ONE 10, e0128265 (2015).

33. Li, S. et al. Causal effect of smoking on DNA methylation in peripheral blood: a twin and family study. Clin. Epigenetics. 10, 18 (2018).

34. Gao, X., Thomsen, H., Zhang, Y., Breitling, L. P. \& Brenner, H. The impact of methylation quantitative trait loci (mQTLs) on active smoking-related DNA methylation changes. Clin. Epigenetics. 9, 87 (2017).

35. Guida, F. et al. Dynamics of smoking-induced genome-wide methylation changes with time since smoking cessation. Hum. Mol. Genet. 24, 2349-2359 (2015).

36. Wilson, R. et al. The dynamics of smoking-related disturbed methylation: a two time-point study of methylation change in smokers, non-smokers and former smokers. BMC Genom. 18, 805 (2017).

37. Shenker, N. S. et al. Epigenome-wide association study in the European Prospective Investigation into Cancer and Nutrition (EPIC-Turin) identifies novel genetic loci associated with smoking. Hum. Mol. Genet. 22, 843-851 (2013).

38. Stueve, T. R. et al. Epigenome-wide analysis of DNA methylation in lung tissue shows concordance with blood studies and identifies tobacco smokeinducible enhancers. Hum. Mol. Genet. 26, 3014-3027 (2017).

39. Ladd-Acosta, C. \& Fallin, M. D. The role of epigenetics in genetic and environmental epidemiology. Epigenomics 8, 271-283 (2016).

40. Birney, E., Smith, G. D. \& Greally, J. M. Epigenome-wide Association Studies and the Interpretation of Disease -Omics. PLoS Genet. 12, e1006105 (2016).

41. Mill, J. \& Heijmans, B. T. From promises to practical strategies in epigenetic epidemiology. Nat. Rev. Genet. 14, 585-594 (2013)

42. Terry, M. B., Delgado-Cruzata, L., Vin-Raviv, N., Wu, H. C. \& Santella, R. M. DNA methylation in white blood cells: association with risk factors in epidemiologic studies. Epigenetics 6, 828-837 (2011).

43. Cook, B. L. et al. Trends in smoking among adults with mental illness and association between mental health treatment and smoking cessation. JAMA 311, 172-182 (2014).

44. Niskanen, L. et al. Inflammation, abdominal obesity, and smoking as predictors of hypertension. Hypertension 44, 859-865 (2004).

45. Dudbridge, F. Power and predictive accuracy of polygenic risk scores. PLoS Genet. 9, e1003348 (2013).

46. Wray, N. R. et al. Research review: Polygenic methods and their application to psychiatric traits. J. Child Psychol. Psychiatry 55, 1068-1087 (2014).

47. Philibert, R. A., Beach, S. R., Lei, M. K. \& Brody, G. H. Changes in DNA methylation at the aryl hydrocarbon receptor repressor may be a new biomarker for smoking. Clin. Epigenetics. 5, 19 (2013).

48. Philibert, R. et al. Reversion of AHRR demethylation is a quantitative biomarker of smoking cessation. Front. Psychiatry 7, 55 (2016).

49. Hannon, E., Lunnon, K. Schalkwyk, L. \& Mill, J. Interindividual methylomic variation across blood, cortex, and cerebellum: implications for epigenetic studies of neurological and neuropsychiatric phenotypes. Epigenetics $\mathbf{1 0}$ 1024-1032 (2015). 